\title{
Volatility spillover between the Korean KOSPI and the Hong Kong HSI stock markets
}

\author{
Eun-Ah Baek $^{a}$, Man-Suk Oh ${ }^{1, a}$ \\ ${ }^{a}$ Department of Statistics, Ewha Womans University, Korea
}

\begin{abstract}
We investigate volatility spillover aspects of realized volatilities (RVs) for the log returns of the Korea Composite Stock Price Index (KOSPI) and the Hang Seng Index (HSI) from 2009-2013. For all RVs, significant long memories and asymmetries are identified. For a model selection, we consider three commonly used time series models as well as three models that incorporate long memory and asymmetry. Taking into account of goodnessof-fit and forecasting ability, Leverage heteroskedastic autoregressive realized volatility (LHAR) model is selected for the given data. The LHAR model finds significant decompositions of the spillover effect from the HSI to the KOSPI into moderate negative daily spillover, positive weekly spillover and positive monthly spillover, and from the KOSPI to the HSI into substantial negative weekly spillover and positive monthly spillover. An interesting result from the analysis is that the daily volatility spillover from the HSI to the KOSPI is significant versus the insignificant daily volatility spillover of the KOSPI to HSI. The daily volatility in Hong Kong affects next day volatility in Korea but the daily volatility in Korea does not affect next day volatility in Hong Kong.
\end{abstract}

Keywords: realized volatility, long memory, asymmetric volatility, high-frequency data, heteroskedasticity, stock market, risk management

\section{Introduction}

The economic integration of international stock markets has grown rapidly over the last two decades. This globalization of stock markets is mainly due to the development of technology and the increased flow of capital between countries. Volatilities, as measured by the standard deviations (or variances of returns) are often used as measures of the total risk of financial assets (Brooks, 2002). Under the internationalization of stock markets, an understanding of volatility spillover (i.e., how the volatilities in stock market of one nation propagates to those of other countries) is important for the application of value at risk, hedging strategies, derivatives pricing, and portfolio diversification.

The availability of high frequency within-day financial transaction data caused French et al. (1987) to consider alternative volatility estimators that are more efficient than those based on daily data. Andersen et al. (2001) called these alternatives realized volatilities. Recent studies on realized volatilities include Andersen et al. (2005, 2011), Deo et al. (2006), Barndorff-Nielsen and Shephard (2007), McAleer and Medeiros (2008), Gonçalves and Meddahi (2009), Chiriac and Voev (2011), Shin and Park (2012) among others.

The advantages of realized volatilities (RVs) over volatilities have prompted studies on realized volatility spillover between financial markets. Recent studies on realized volatility spillover focused

\footnotetext{
${ }^{1}$ Corresponding author: Department of Statistics, Ewha Womans University, 52, Ewhayeodae-gil, Seodaemun-gu, Seoul 03760, Korea. E-mail: msoh@ewha.ac.kr
}

Published 31 May 2016/ journal homepage: http://csam.or.kr

(c) 2016 The Korean Statistical Society, and Korean International Statistical Society. All rights reserved. 
on Asian stock markets are Park and Shin (2014) which studied RV spillover between the S\&P 500 index and the Korea Composite Stock Price Index (KOSPI) and Jung and Maderitsch (2014) which studied RV spillover among the Hong Kong Hang Seng Index (HSI), the Euro Stoxx 50 and the S\&P 500 .

In this paper, we investigate economic linkage between Korea and China via RV spillover between the Hong Kong HSI and the KOSPI. We first investigate long memory and asymmetry of RV for the KOSPI and HSI through unit root tests and sample autocorrelation functions on log returns, RVs, and $\log$ prices. The log returns, RVs, and the log prices are shown to have different long range dependence in that the log returns have short memories, RVs are stationary with long memories, and the log prices are non-stationary with long memories. It is also revealed that RVs have asymmetry property in that the positive log returns for the KOSPI and the HSI have more persistent memories than the negative counterparts.

Based on the above analysis, we then perform model selection for the RVs of the log returns. We consider three commonly used time series models as well as three models incorporating long memory and asymmetry as candidate models. The best model among the six candidate models is selected based on model selection criteria such as prediction errors and goodness-of-fit. A leverage heteroskedastic autoregressive realized volatility (LHAR) model is selected as the best model for the given data in terms of volatility forecast since it produces superior forecasts in the KOSPI and the HSI.

Analysis based on the selected LHAR model reveals significant decompositions of the spillover effects from the HSI to the KOSPI into moderate negative daily spillover, positive weekly spillover and positive monthly spillover, and that from the KOSPI to the HSI into substantial negative weekly spillover and positive monthly spillover. The most interesting finding from the analysis is that the daily RV spillover from the HSI to the KOSPI is significant versus the insignificant daily RV spillover of the KOSPI to the HSI. The daily volatility in Hong Kong affects the next day volatility in Korea but the daily volatility in Korea does not affect next day volatility in Hong Kong.

The rest of this paper is organized as follows. Basic analysis of the KOSPI and the HSI is done in Section 2, and the model selection for the given data among six forecast models is done in Section 3. We analyze the realized volatility spillover effects between Korea and Hong Kong in Section 4. The last section provides a summary and discussion.

\section{Data analysis}

We consider stock market data of the KOSPI and the HSI from 2009 to 2013. One-minute price data sets of the KOSPI is purchased from the Korea Exchange (www.krx.co.kr) and that of the HSI is purchased from Hang Seng Indexes Company (www.hsi.com.hk/HSI-Net/HSI-Net).

We begin the analysis with a simple visual inspection of the daily closing prices of the two assets (Figure 1). This figure shows that prices of the assets are tending upwards in a broad perspective. The prices reached the lowest point in 2009, plunged in late 2011, but increased again after 2011. Both the KOSPI and the HSI daily prices seem to have a similar pattern.

Figure 2 presents the log returns of the two assets. It shows spread around 0 and significant volatility clustering. The basic statistics of the log returns are summarized in Table 1 . From these preliminary observations, the two stock market series seem to show similar patterns.

The RV is defined as follows. Assume that a basic asset is given. Let $p_{t}=\ln P_{t}$ be the $\log$ of the ending price $P_{t}$ of the asset at day $t$. Let $r_{t}=p_{t}-p_{t-1}$ be the daily log return. We assume that for each working day the $\mathrm{M}$ equal-interval $\log$ return data, $r_{t-j \Delta}=p_{t-j \Delta}-p_{t-(j-1) \Delta}, j=1,2, \ldots, M$, is available for the working time, where $\Delta=1 / M$ is the given sampling interval. The daily RV is now 


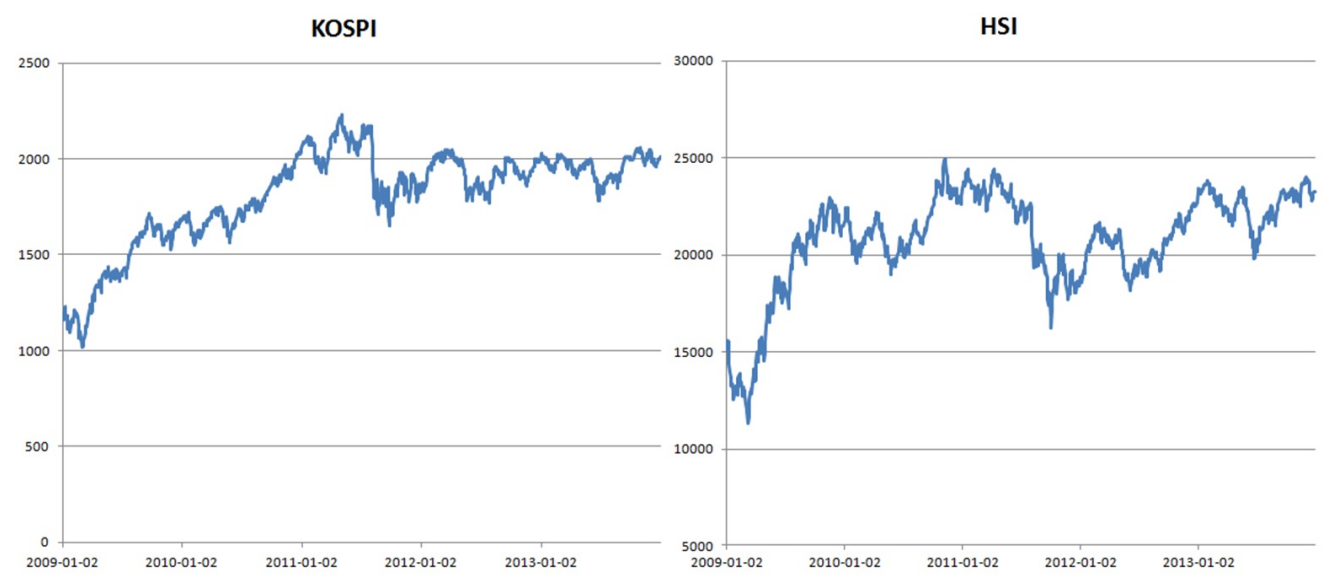

Figure 1: Daily prices of the Korea Composite Stock Price Index (KOSPI) and the Hong Kong Hang Seng Index (HSI).

KOSPI

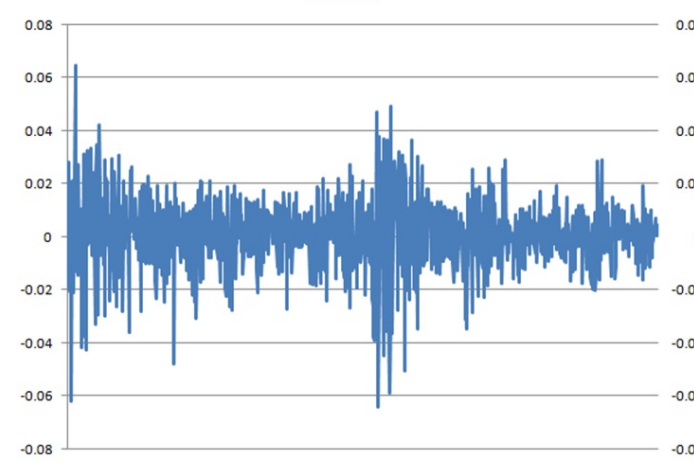

HSI

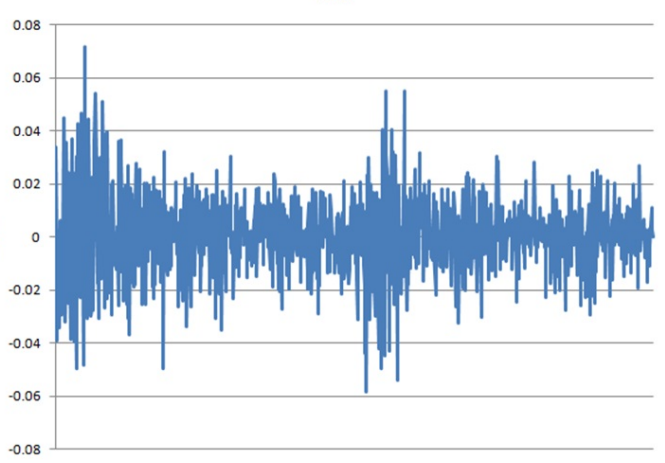

Figure 2: Daily log-returns of the Korea Composite Stock Price Index (KOSPI) and the Hong Kong Hang Seng Index (HSI).

Table 1: Descriptive statistics of daily log-returns of KOSPI and HSI

\begin{tabular}{ccccccrr}
\hline \hline & Mean & Median & Min & Max & SD & Skew & Kurt \\
\hline KOSPI & 0.00042 & 0.00047 & -0.0642 & 0.0648 & 0.0125 & -0.33 & 3.18 \\
HSI & 0.00036 & 0.00051 & -0.0583 & 0.0715 & 0.0144 & 0.01 & 1.81 \\
\hline \hline KOSPI = Kor
\end{tabular}

KOSPI = Korea Composite Stock Price Index, HSI = Hong Kong Hang Seng Index.

defined by

$$
\mathrm{RV}_{t}^{(d)}=\sqrt{\sum_{j=1}^{M} r_{t-j \Delta}^{2}}
$$

$\mathrm{RV}$ s are computed using a $\Delta=1$ minute within-day sample and are displayed in Figure 3. We have chosen the one-minute sampling interval based on the recommendations of Bandi and Russell (2008) and Oh and Shin (2012). The value of RVs are extremely large in early 2009 when both the KOSPI and the HSI reached the lowest points as well as in late 2011 when they plunged. 


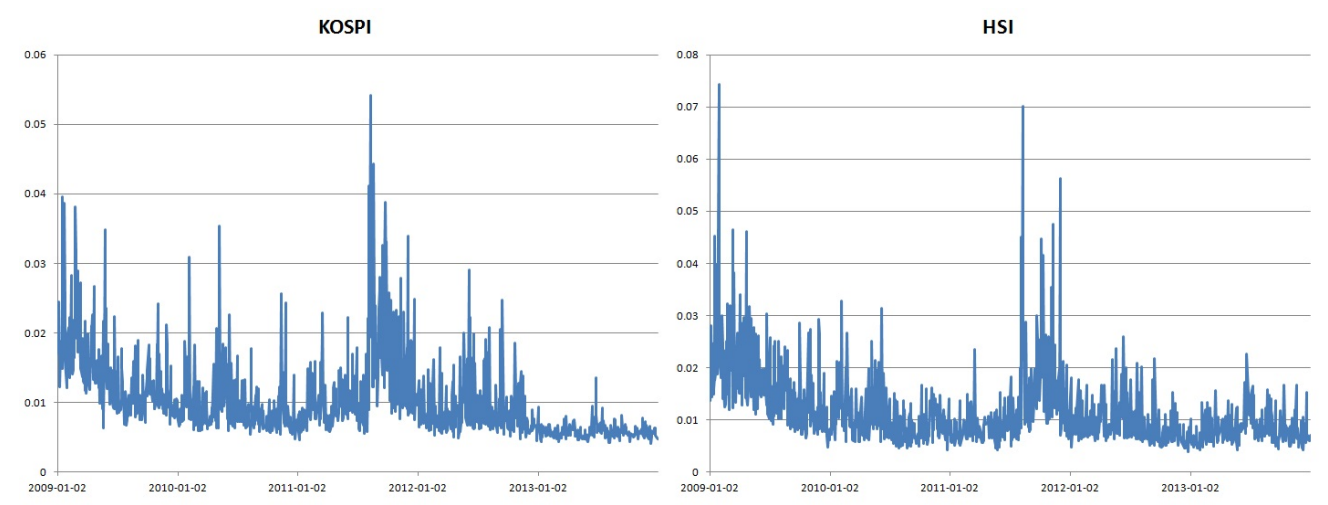

Figure 3: Realized volatility of the Korea Composite Stock Price Index (KOSPI) and the Hong Kong Hang Seng Index (HSI).

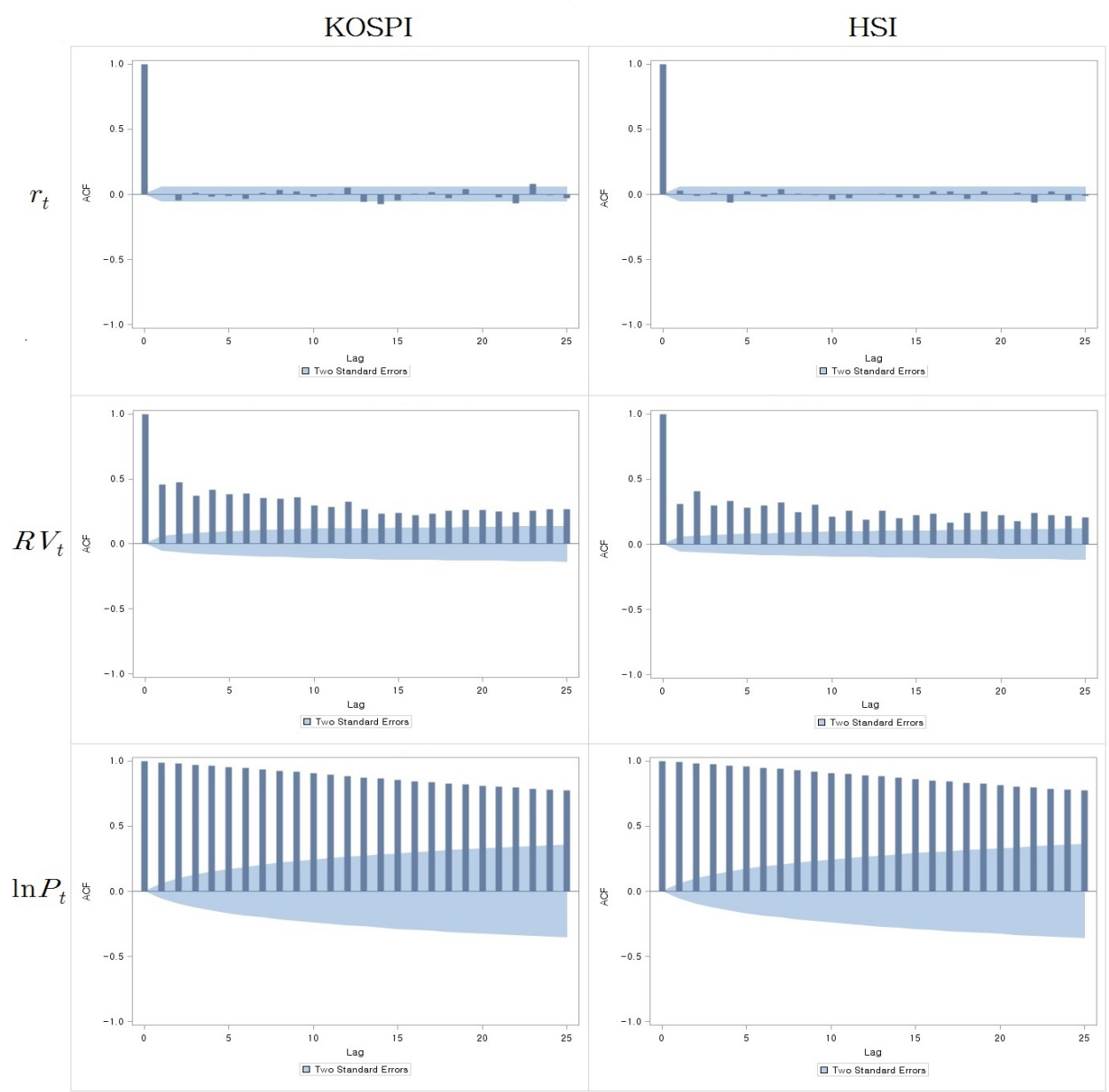

Figure 4: Sample autocorrelation functions $(A C F s)$ of the log-return, the realized volatilities $(R V s)$, and the log-price of the Korea Composite Stock Price Index (KOSPI) and the Hong Kong Hang Seng Index (HSI). 
Table 2: Augmented Dickey-Fuller (ADF) tests for unit root of realized volatility and daily log prices of KOSPI and HSI

\begin{tabular}{|c|c|c|c|c|c|c|}
\hline & \multicolumn{3}{|c|}{$\overline{\mathrm{RV}_{t}}$} & \multicolumn{3}{|c|}{$\overline{\ln P_{t}}$} \\
\hline & lag & ADF test & $p$-value & lag & ADF test & $p$-value \\
\hline KOSPI & 10 & -3.78 & 0.0058 & 1 & -2.78 & 0.2130 \\
\hline HSI & 9 & -4.92 & 0.0001 & 1 & -2.32 & 0.1655 \\
\hline
\end{tabular}

KOSPI = Korea Composite Stock Price Index, HSI = Hong Kong Hang Seng Index.

KOSPI
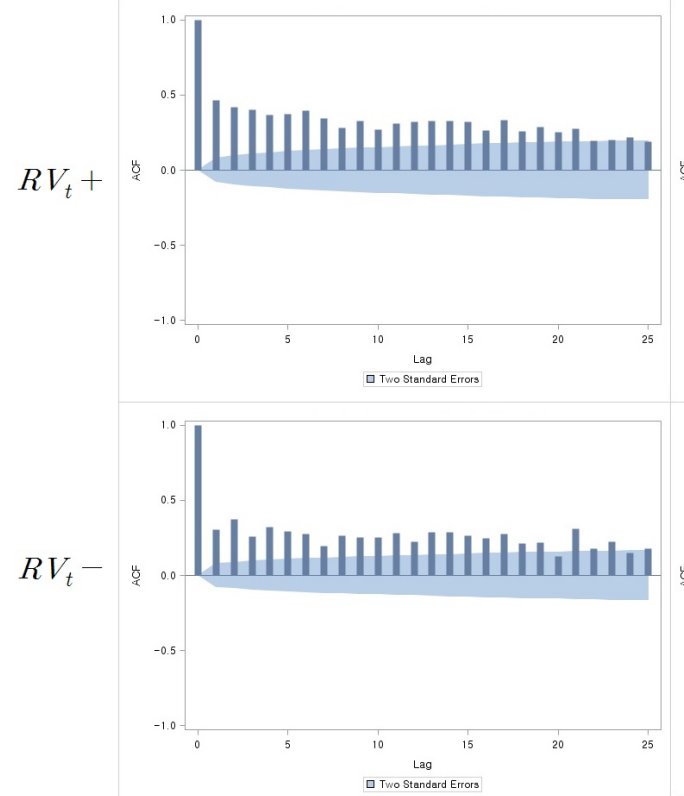

HSI
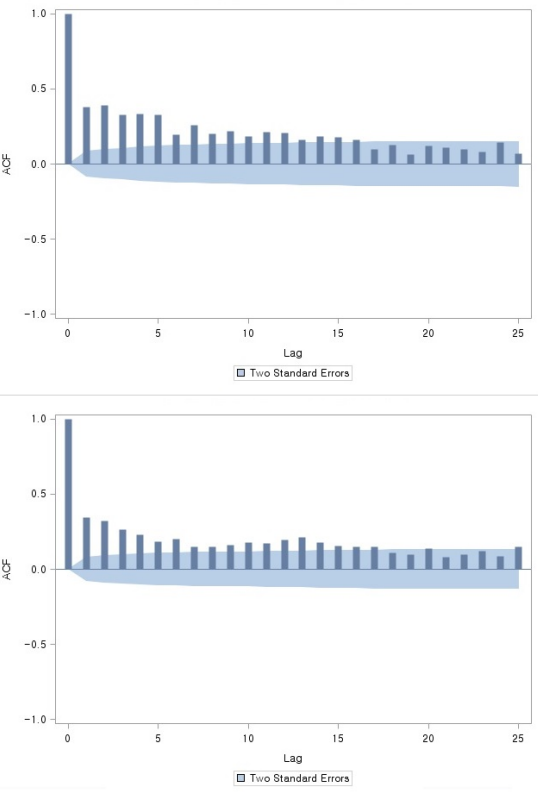

Figure 5: Sample autocorrelation functions (ACFs) of the realized volatilities (RVs) for positive return and negative return.

Now we investigate basic long memory properties via unit root tests and sample autocorrelation functions of the asset log prices, log returns, and RVs. First, basic memory properties are displayed in the sample autocorrelation functions (ACFs) in Figure 4. The ACF plot of the daily log return shows a pattern close to that of white noise and implies that the daily log return has little memory. In contrast, the RV has more persistent memory and the log price has the most persistent memory.

Table 2 provides the augmented Dickey-Fuller (ADF) $t$-test results on RV and $p_{t}=\ln P_{t}$. Mean models and AR lags, selected by the Akaike Information Criterion (AIC), are used. Table 2 shows significant evidence of unit root for the log price but not for the RVs. This reveals that the long memory of the RVs is not a consequence of the unit root while that of the log price is. The rejected unit root tests for the RVs indicate that the data-generating processes for the RVs are stationary even though they demonstrate long memory. This result is consistent with the observations obtained from the ACF plots.

Asymmetry of the RVs is studied by splitting them into two terms corresponding to positive returns and negative returns. The series of the positive part $\mathrm{RV}_{t}+$ is constructed from the RV series by deleting 
terms that correspond to the negative returns. The negative part $\mathrm{RV}_{t}-$ is constructed similarly. To understand the existence of asymmetry, plots of the sample ACFs of these two series for each of the KOSPI and the HSI are provided in Figure 5. The sample ACFs are obtained as if $\mathrm{RV}_{t}+\left(\mathrm{RV}_{t}-\right)$ were regularly spaced time series. It reveals that the positive and the negative series have different patterns in that the positive series have more persistent memory than the negative series for both the KOSPI and the HSI. Therefore, analysis of the given data incorporating the asymmetry property would be appropriate.

\section{Model selection}

As candidate models for the given stock market data, we first consider three classical stationary autoregression models, $\mathrm{AR}(1), \mathrm{AR}(3), \mathrm{AR}(22)$. $\mathrm{AR}(1)$ is the simplest form of time-varying processes. $\mathrm{AR}(3)$ and $\mathrm{AR}(22)$ are chosen since three days and monthly data with 22 business days are often used to analyze stock data. We also consider three versions of HAR-RV (heteroskedastic autoregressive realized volatility) models; HAR-RV, LHAR-RV, THAR-RV. These three models incorporate long memories and asymmetries of the asset returns in terms of RV spillovers. The original HAR-RV model is introduced by Corsi (2009). It captures the persistent heteroskedastic volatilities by considering daily, weekly, and monthly moving averages of RVs as regressors. LHAR-RV model added an asymmetry feature to the HAR-RV model by adding leverage terms (McAleer and Medeiros, 2008; Corsi and Reno, 2009). THAR-RV model (threshold HAR-RV) is also a modified version of HAR-RV model which directly incorporates the asymmetry features. It is introduced by Park and Shin (2014) and showed good volatility forecasting performance in some Korean assets. We suppress ' $-\mathrm{RV}$ ' in the above HAR models from here on for ease of exposition.

To investigate the dynamic volatility spillover effects between the KOSPI and the HSI, we consider modifications of the six models. Let $\mathrm{RV}_{1, t}$ be the RV of either the HSI or the KOSPI and $\mathrm{RV}_{2, t}$ be that of the other. The Subscript 1 and 2 of $\beta^{(d)}, \beta^{(w)}, \beta^{(m)}, r^{(d)}, r^{(w)}, r^{(m)}$ are defined in the same way. The modified models are given as

$$
\begin{aligned}
\operatorname{AR}(1): \mathrm{RV}_{1, t+1}= & \beta_{0}+\beta_{1} \mathrm{RV}_{1, t}+\beta_{2} \mathrm{RV}_{2, t}+\alpha_{t+1}, \\
\operatorname{AR}(3): \mathrm{RV}_{1, t+1}= & \beta_{0}+\sum_{i=1}^{3} \beta_{i} \mathrm{RV}_{1, t+1-i}+\sum_{j=1}^{3} \beta_{j+3} \mathrm{RV}_{2, t+1-j}+\alpha_{t+1}, \\
\operatorname{AR}(22): \mathrm{RV}_{1, t+1}= & \beta_{0}+\sum_{i=1}^{22} \beta_{i} \mathrm{RV}_{1, t+1-i}+\sum_{j=1}^{22} \beta_{j+22} \mathrm{RV}_{2, t+1-j}+\alpha_{t+1}, \\
\operatorname{HAR}(3): \mathrm{RV}_{1, t+1}= & \beta_{0}+\beta_{1}^{(d)} \mathrm{RV}_{1, t}^{(d)}+\beta_{1}^{(w)} \mathrm{RV}_{1, t}^{(w)}+\beta_{1}^{(m)} \mathrm{RV}_{1, t}^{(m)}+\beta_{2}^{(d)} \mathrm{RV}_{2, t}^{(d)}+\beta_{2}^{(w)} \mathrm{RV}_{2, t}^{(w)} \\
& +\beta_{2}^{(m)} \mathrm{RV}_{2, t}^{(m)}+\alpha_{t+1}, \\
\operatorname{LHAR}(3): \mathrm{RV}_{1, t+1}^{(d)}= & \beta^{0}+\beta_{1}^{(d)} \mathrm{RV}_{1, t}^{(d)}+\beta_{1}^{(w)} \mathrm{RV}_{1, t}^{(w)}+\beta_{1}^{(m)} \mathrm{RV}_{1, t}^{(m)}+\gamma_{1}^{(d)} r_{1, t}^{(d)-}+\gamma_{1}^{(w)} r_{1, t}^{(w)-}+\gamma_{1}^{(m)} r_{1, t}^{(m)-} \\
& +\beta_{2}^{(d)} \mathrm{RV}_{2, t}^{(d)}+\beta_{2}^{(w)} \mathrm{RV}_{2, t}^{(w)}+\beta_{2}^{(m)} \mathrm{RV}_{2, t}^{(m)}+\gamma_{2}^{(d)} r_{2, t}^{(d)-}+\gamma_{2}^{(w)} r_{2, t}^{(w)-}+\gamma_{2}^{(m)} r_{2, t}^{(m)-}+\alpha_{t+1}, \\
\operatorname{THAR}(3): \mathrm{RV}_{1, t+1}^{(d)}= & \beta_{k}^{0}+\sum_{k=1}^{2}\left(\beta_{1, k}^{(d)} \mathrm{RV}_{1, t}^{(d)}+\beta_{1, k}^{(w)} \mathrm{RV}_{1, t}^{(w)}+\beta_{1, k}^{(m)} \mathrm{RV}_{1, t}^{(m)}\right) I_{k 1 t} \\
& +\sum_{k=1}^{2}\left(\beta_{2, k}^{(d)} \mathrm{RV}_{2, t}^{(d)}+\beta_{2, k}^{(w)} \mathrm{RV}_{2, t}^{(w)}+\beta_{2, k}^{(m)} \mathrm{RV}_{2, t}^{(m)}\right) I_{k 2 t}+\alpha_{t+1},
\end{aligned}
$$


Table 3: Model selection criteria for the modified models

\begin{tabular}{cccccccc}
\hline \hline Model & & $100 \times$ MAE & MAPE & $100 \times$ RMSE & $R^{2}$ & AIC & BIC \\
\hline & AR(1) & 0.3196 & 31.754 & 0.4845 & 0.3005 & -9377.91 & -9357.55 \\
& AR(3) & 0.2880 & 27.448 & 0.4428 & 0.4133 & $\mathbf{- 9 5 6 9 . 8 7}$ & $\mathbf{- 9 5 2 9 . 1 6}$ \\
\multirow{5}{*}{ HSI to KOSPI } & AR(22) & $\mathbf{0 . 2 6 5 7}$ & $\mathbf{2 5 . 2 2 2}$ & $\mathbf{0 . 4 0 6 9}$ & $\mathbf{0 . 4 7 7 2}$ & -9541.33 & -9307.99 \\
& HAR(3) & 0.2776 & 26.800 & 0.4311 & 0.4044 & -9478.26 & -9437.67 \\
& LHAR(3) & 0.2736 & 26.416 & 0.4192 & 0.4447 & -9545.14 & -9479.19 \\
& THAR(3) & 0.2777 & 26.787 & 0.4246 & 0.4315 & -9521.19 & -9450.15 \\
\hline \multirow{6}{*}{ KOSPI to HSI } & AR(1) & 0.4054 & 38.251 & 0.6150 & 0.1887 & -8805.67 & -8785.31 \\
& AR(3) & 0.3648 & 33.339 & 0.5562 & 0.3341 & $\mathbf{- 9 0 2 3 . 6 7}$ & -8982.97 \\
& AR(22) & $\mathbf{0 . 3 2 6 9}$ & 30.538 & $\mathbf{0 . 4 8 6 1}$ & $\mathbf{0 . 4 1 9 9}$ & $\mathbf{- 9 1 2 2 . 1 4}$ & -8888.81 \\
& HAR(3) & 0.3367 & 31.091 & 0.5097 & 0.3641 & $\mathbf{- 9 0 9 8 . 5 4}$ & $\mathbf{- 9 0 6 8 . 0 9}$ \\
& LHAR(3) & 0.3287 & $\mathbf{3 0 . 1 4 7}$ & 0.5014 & 0.3841 & $\mathbf{- 9 1 2 1 . 5 2}$ & -9050.49 \\
& THAR(3) & 0.3342 & 30.785 & 0.5049 & 0.3761 & $\mathbf{- 9 1 1 2 . 2 8}$ & -9041.24 \\
\hline \hline
\end{tabular}

MAE = mean absolute error, MAPE = mean absolute percentage error, RMSE = root mean square error, AIC = Akaike Information Criterion, BIC $=$ Bayesian Information Criterion, KOSPI $=$ Korea Composite Stock Price Index, HSI $=$ Hong Kong Hang Seng Index.

where $\alpha_{t}$ is an error sequence, $r^{(d)-}, r^{(w)-}, r^{(m)-}$ are the negative part of the daily, weekly, monthly $\log$ returns, respectively, $\mathrm{RV}_{t}^{(w)}, \mathrm{RV}_{t}^{(m)}$ are the one-week moving average and the one-month moving average, respectively, of the daily $\mathrm{RV}_{t}^{(d)}$, given by

$$
\begin{aligned}
& \mathrm{RV}_{t}^{(w)}=\frac{\mathrm{RV}_{t}+\mathrm{RV}_{t-1}+\mathrm{RV}_{t-2}+\mathrm{RV}_{t-3}+\mathrm{RV}_{t-4}}{5}, \\
& \mathrm{RV}_{t}^{(m)}=\frac{\mathrm{RV}_{t}+\mathrm{RV}_{t-1}+\mathrm{RV}_{t-2}+\mathrm{RV}_{t-3}+\mathrm{RV}_{t-21}}{22},
\end{aligned}
$$

and in THAR(3), $I_{1 t}=I\left(r_{t} 0\right), I_{2 t}=1-I_{1 t}$ are the indicator functions of positive and negative return, respectively. Note that in these modified models, additional terms contain RVs of the other market index.

Good forecasting ability is important for this kind of data; consequently, we consider several other measures of forecasting performance in model selection in addition to traditional model selection criteria such as BIC and AIC. For each model, a one-step-ahead pseudo forecast $\widehat{\mathrm{RV}}_{t+1 \mid t}$ is computed. The forecasting performances are evaluated based on mean absolute error (MAE), mean absolute percentage error (MAPE) and root mean square error (RMSE), where

$$
\begin{aligned}
\text { MAE } & =\frac{1}{n} \sum_{t=1}^{n}\left|\mathrm{RV}_{t+1}-\widehat{\mathrm{RV}}_{t+1 \mid t}\right|, \\
\text { MAPE } & =\frac{1}{n} \sum_{t=1}^{n} \frac{\left|\mathrm{RV}_{t+1}-\widehat{\mathrm{RV}}_{t+1 \mid t}\right|}{\mathrm{RV}_{t+1}} \times 100 \%, \\
\text { RMSE } & =\sqrt{\frac{1}{n} \sum_{t=1}^{n}\left(\mathrm{RV}_{t+1}-\widehat{\mathrm{RV}}_{t+1 \mid t}\right)^{2} .}
\end{aligned}
$$

Expectation power of models is calculated by MAE, MAPE, RMSE, explanation power is measured by $R^{2}$ and goodness-of-fit is calculated by AIC, BIC.

The selection criteria in Table 3 take into account of expectation power, explanation power and goodness-of-fit of models in different ways, and no single model is dominant. AR(22) from the HSI to the KOSPI and AR(22) and LHAR(3) from the KOSPI to the HSI have higher power of expectation. 

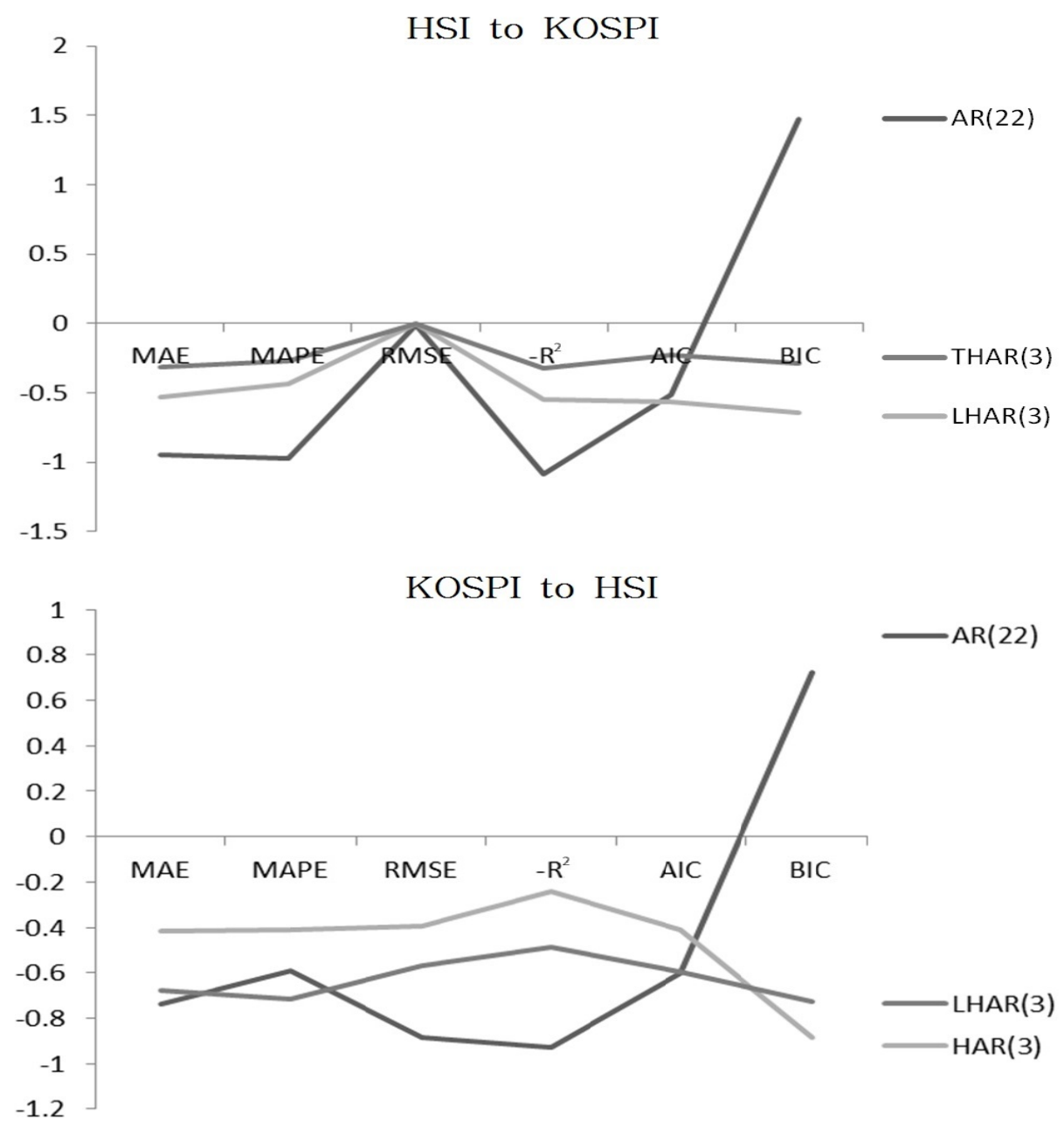

Figure 6: Model selection criteria (MAE = mean absolute error, $M A P E=$ mean absolute percentage error, $R M S E$ $=$ root mean square error, $A I C=$ Akaike Information Criterion, $B I C=$ Bayesian Information Criterion, $K O S P I=$ Korea Composite Stock Price Index, HSI = Hong Kong Hang Seng Index).

$\mathrm{AR}(22)$ has higher power of explanation both from the HSI to the KOSPI and from the KOSPI to the HSI. AR(3) from the HSI to the KOSPI, and AR(22) and HAR(3) from the KOSPI to the HSI have better goodness-of-fit (taking into account the number of parameters).

To select a good model considering all these criteria, we standardize the criteria and visualize them for the best three models (Figure 6). The lower the value of the criterion, the better the performance of a model, except for $R^{2}$. Thus, for easy visual comparison we plot the standardized $-R^{2}$ instead of $R^{2}$. Figure 6 shows that $\mathrm{AR}(22)$ has good expectation power and goodness-of-fit but a very large BIC value as a result of too many parameters. The AICs of the AR(22) and the LHAR(3) are very close in both plots, while on the basis of the BIC (which imposes larger penalty for additional coefficients), the LHAR(3) is clearly preferred to the AR(22). We choose LHAR(3) as a final model for analysis of volatility spillover between the KOSPI and the HSI since it seems to have the best overall performance. 
Table 4: Estimation results of the LHAR(3)

\begin{tabular}{ccc}
\hline & HSI to KOSPI & KOSPI to HSI \\
\hline$\beta^{0}$ & $0.0016(0.0004)^{* * *}$ & $0.0011(0.0004) *$ \\
$\beta_{1}^{(d)}$ & $3.5553(1.0807)^{* *}$ & $-0.0386(0.0400)$ \\
$\beta_{1}^{(w)}$ & $-6.0585(1.4630)^{* * *}$ & $0.3328(0.0724)^{* * *}$ \\
$\beta_{1}^{(m)}$ & $2.8712(0.5436)^{* * *}$ & $0.5828(0.0709)^{* * *}$ \\
$\gamma_{1}^{(d)}$ & $-0.0390(0.0154)^{*}$ & $-0.0252(0.0155)$ \\
$\gamma_{1}^{(w)}$ & $-0.0733(0.0406)$ & $-0.0959(0.0404) *$ \\
$\gamma_{1}^{(m)}$ & $-0.1924(0.0807)^{*}$ & $0.0507(0.0809)$ \\
$\beta_{2}^{(d)}$ & $-0.1651(0.0334)^{* * *}$ & $1.4714(1.2927)$ \\
$\beta_{2}^{(w)}$ & $0.1860(0.0605)^{* *}$ & $-3.7826(1.7499)^{*}$ \\
$\beta_{2}^{(m)}$ & $0.4326(0.0592)^{* * *}$ & $2.3642(0.6502)^{* * *}$ \\
$\gamma_{2}^{(d)}$ & $-0.0132(0.0130)$ & $0.0166(0.0185)$ \\
$\gamma_{2}^{(w)}$ & $-0.0557(0.0338)$ & $-0.0808(0.0486)$. \\
$\gamma_{2}^{(m)}$ & $0.0627(0.0676)$ & $-0.0214(0.0965)$
\end{tabular}

KOSPI $=$ Korea Composite Stock Price Index, HSI $=$ Hong Kong Hang Seng Index.

\section{Realized volatility spillover}

Table 4 shows the LHAR(3) regression results. The estimates marked with '. ', ‘*', ‘**', ‘***' are significant with levels $10 \%, 5 \%, 1 \%, 0.01 \%$, respectively.

We first investigate RV spillover effects from the HSI to the KOSPI. The effects of the daily, weekly and monthly RVs of the KOSPI and the HSI on the next days RVs of the KOSPI are significant. The estimated coefficients of the daily, weekly and monthly RVs of the KOSPI are 3.5553, -6.0585 and 2.8712 respectively, while those of the HSI are $-0.1651,0.1860$ and 0.4326 , respectively. Note that the daily, weekly, and monthly RVs of the KOSPI and the HSI are all significant but have different effectiveness. The estimated asymmetry parameters show that the negative daily and weekly log returns of both the KOSPI and the HSI all increase the next day RVs of the KOSPI. However, the monthly log returns of the KOSPI increase the next day RVs of the KOSPI but the monthly log returns of the HSI decrease the next day RVs of the KOSPI. The daily and monthly log returns of the KOSPI are significant and imply that the RVs for negative daily/monthly return days are more persistent than those for positive daily/monthly return days in the Korean stock market.

We next investigate the volatility spillover from the KOSPI to the HSI. The estimated coefficients of the daily, weekly and monthly coefficients of the HSI are $-0.0386,0.3328$ and 0.5828 , respectively. Those of the KOSPI are $1.4174,-3.7826$ and 2.3642 , respectively. The weekly and monthly RVs of the KOSPI and the HSI are all significant; however, the daily RVs of the KOSPI and the HSI are not. The estimated asymmetry parameters show that the negative daily and weekly log returns of the HSI decreases the next day RVs of the HSI but the negative monthly log returns of the HSI increases the next day RVs of the HSI. The negative daily log returns of the KOSPI increase the next day RVs of the HSI but the negative weekly and monthly log returns of the KOSPI decrease the next day RVs of the HSI. The weekly log return of the HSI is significant at 5\% level. It shows that the RV for a negative weekly returns day in the Hong Kong stock market is more persistent than the positive weekly returns in the Hong Kong stock market.

We summarize the analysis results. The daily volatility spillover from the HSI to the KOSPI is significant versus the insignificant daily volatility spillover of the KOSPI to the HSI. The daily volatility in Hong Kong affects the next day volatility in Korea but the daily volatility in Korea does not affect the next day volatility in Hong Kong. The weekly and monthly volatilities both from the HSI to the KOSPI and from the KOSPI to the HSI are all significant, but have different signals. The 
estimated coefficient for the weekly volatility from the HSI to the KOSPI is positive but that from the KOSPI to the HSI is negative. The estimated coefficients for the monthly volatility spillovers from the HSI to the KOSPI and those from the KOSPI to the HSI are positive.

Looking at the estimated significant volatility spillover coefficients, high daily volatility of the HSI result in substantial decrease in the next day volatility in the KOSPI due to negative daily spillover in the HSI, which is counteracted by moderately increased volatilities due to the positive weekly and monthly volatility spillover. However, the high weekly volatility of the KOSPI substantially decrease the next day volatility in the HSI due to the negative weekly spillover, which is counteracted by a substantially increased volatility due to the positive monthly volatility spillover.

\section{Conclusion}

The daily volatility in Korea does not significantly affect Hong Kong on the next day, but the daily volatility spillover from Hong Kong to Korea is significant and negative. Large volatility of the HSI decreases the next day volatility of the KOSPI. Weekly volatility spillovers both from the KOSPI to the HSI and from the HSI to the KOSPI are significant but have different signals. It positive for the HSI to the KOSPI but negative for the KOSPI to the HSI. Monthly volatility spillovers both from the KOSPI to the HSI and from the HSI to the KOSPI are significant and are positive.

The Hu-Gang Tong or Shanghai-Hong Kong stock connect was launched on November 17, 2014. Hu-Gang Tong allows investors in Hong Kong and on the mainland to trade a rage of stocks listed on the other side's bourse through securities firms in their own market; consequently, there is a need for further study about volatility spillover before and after Hu-Gang Tong between the KOSPI and the HSI.

\section{Acknowledgements}

This research was supported by the Basic Science Research Program through the National Research Foundation of Korea (NRF) funded by the Ministry of Education, Science and Technology (2013R1A1 A2005481).

\section{References}

Andersen TG, Bollerslev T, Diebold FX, and Labys P (2001). The distribution of realized volatility exchange rate volatility, Journal of the American Statistical Association, 96, 42-55.

Andersen TG, Bollerslev T, and Meddahi N (2005). Correcting the errors: volatility forecast evaluation using high-frequency data and realized volatilities, Econometrica, 73, 279-296.

Andersen TG, Bollerslev T, and Meddahi N (2011). Realized volatility forecasting and market microstructure noise, Journal of Econometrics, 160, 220-234.

Bandi FM and Russell JR (2008). Microstructure noise, realized variance, and optimal sampling, Review of Economic Studies, 75, 339-369.

Barndorff-Nielsen OE and Shephard N (2007). Variation, jumps, market frictions and high frequency data in financial econometrics. In R. Blundell, W. Newey, and T. Persson (Eds), Advances in Economics and Econometrics: Theory and Application (pp. 328-372), Cambridge University Press, Cambridge.

Brooks C (2002). Introductory Econometrics for Finance, Cambridge University Press, Cambridge.

Chiriac R and Voev V (2011). Modelling and forecasting multivariate realized volatility, Journal of Applied Econometrics, 26, 922-947. 
Corsi F (2009). A simple approximate long-memory model of realized volatility, Journal of Financial Econometrics, 7, 174-196.

Corsi F and Reno R (2009). HAR volatility modelling with heterogeneous leverage and jumps, Retrieved April 30, 2016, http://ecares.ulb.ac.be/ecaresdocuments/seminars0910/corsi2.pdf

Deo R, Hurvich C, and Lu Y (2006). Forecasting realized volatility using a long-memory stochastic volatility model: estimation, prediction and seasonal adjustment, Journal of Econometrics, 131, $29-58$.

French KR, Schwert GW, and Stambaugh RF (1987). Expected stock returns and volatility, Journal of Financial Economics, 19, 3-29.

Gonçalves S and Meddahi N (2009). Bootstrapping realized volatility, Econometrica, 77, 283-306.

Jung RC and Maderitsch R (2014). Structural breaks in volatility spillovers between international financial markets: contagion or mere interdependence?, Journal of Banking and Finance, 47, 331-342.

McAleer M and Medeiros MC (2008). Realized volatility: a review, Econometric Reviews, 27, 10-45.

Park S and Shin DW (2014). Modeling and forecasting realized volatilities of Korean financial assets featuring long memory and asymmetry, Asia-Pacific Journal of Financial Studies, 43, 31-58.

Oh R and Shin DW (2012). Market microstructure noise and optimal sampling frequencies for the realized variances of stock prices of four leading Korean companies, Korean Journal of Applied Statistics, 25, 15-27.

Shin DW and Park S (2012). Efficient realized variance, regression coefficient, and correlation coefficient under different sampling frequencies, Economics Letters, 115, 334-337. 